\title{
Dense molecular clouds and associated star formation in the Magellanic Clouds
}

\author{
Mónica Rubio ${ }^{1}$ \\ ${ }^{1}$ Departamento de Astronomia, Universidad de Chile, Casilla 36-D, Santiago, Chile \\ email: mrubio@das.uchile.cl
}

\begin{abstract}
Multiwavelengths studies of massive star formation regions in the LMC and SMC reveal that a second generation of stars is being formed in dense molecular clouds located in the surroundings of the massive clusters. These dense molecular clouds have survived the action of massive star UV radiation fields and winds and they appear as compact dense $\mathrm{H}_{2}$ knots in regions of weak $\mathrm{CO}$ emission. Alternatively, we have found that large molecular clouds, probably remnants of the parental giant molecular clouds where the first generation of stars were formed, are suffering the interaction of the winds and UV radiation field in their surfaces in the direction of the central massive cluster or massive stars. These molecular regions show $1.2 \mathrm{~mm}$ continuum emission form cold dust and they show embedded IR sources as determined from deep ground base JHKs imaging. The distribution of young IR sources as determined from their Mid IR colors obtained by SPITZER concentrate in the maxima of CO and dust emission. IR spectroscopy of the embedded sources with high IR excess confirm their nature as massive young stellar objects (MYSO's). Our results are suggestive of contagious star formation where triggering and induced star formation could be taking place.
\end{abstract}

Keywords. Magellanic Clouds, Star Formation, Molecular Clouds

\section{Introduction}

Massive Star Formation occurs in giant molecular clouds. In the Magellanic Clouds $\mathrm{CO}(1-0)$ surveys have traced the distribution if giant molecular clouds (Fukui et al. 1999; Mizuno et al. 2001). In these galaxies, the ISM has different conditions such as a lower dust to gas ratio, and thus the shielding of molecules from the external UV radiation field is not as effective as in our Galaxy. Moreover, the amount of gas in GMC's remain unknown since $\mathrm{H}_{2}$ is almost impossible to observe directly in cold interstellar regions and $\mathrm{CO}$ seems to be present only in the densest part of the molecular clouds. Thus, we are faced with the following questions. Is the CO a reliable tracer of the molecular gas? Are the properties of the CO clouds similar or different to those in our Galaxy? How do these issues affect the determination of the molecular mass? These questions are further complicated by the fact that the $\mathrm{C}$ and $\mathrm{O}$ elements are less abundant and thus $\mathrm{CO}$ is more difficult to form. The CO observations reveal that the emission in the Magellanic Clouds is very weak and thus difficult to observe.

We have gathered a large set of observations done with different telescopes and wavelengths (VLT, NTT, LCO, CTIO, ISO, SEST, SIMBA, HST) and we have found evidence of a second generation of massive stars in several of the massive star forming regions in the LMC and SMC. We have investigated their embedded stellar content in the molecular regions by deep NIR imaging, and we are currently doing a spectroscopic follow up of the brightest embedded IR sources detected. In addition, we are doing studies using the millimeter continuum emission obtained with the SIMBA bolometer as an alternative way to determine the amount of the molecular gas. We are combining these data with 


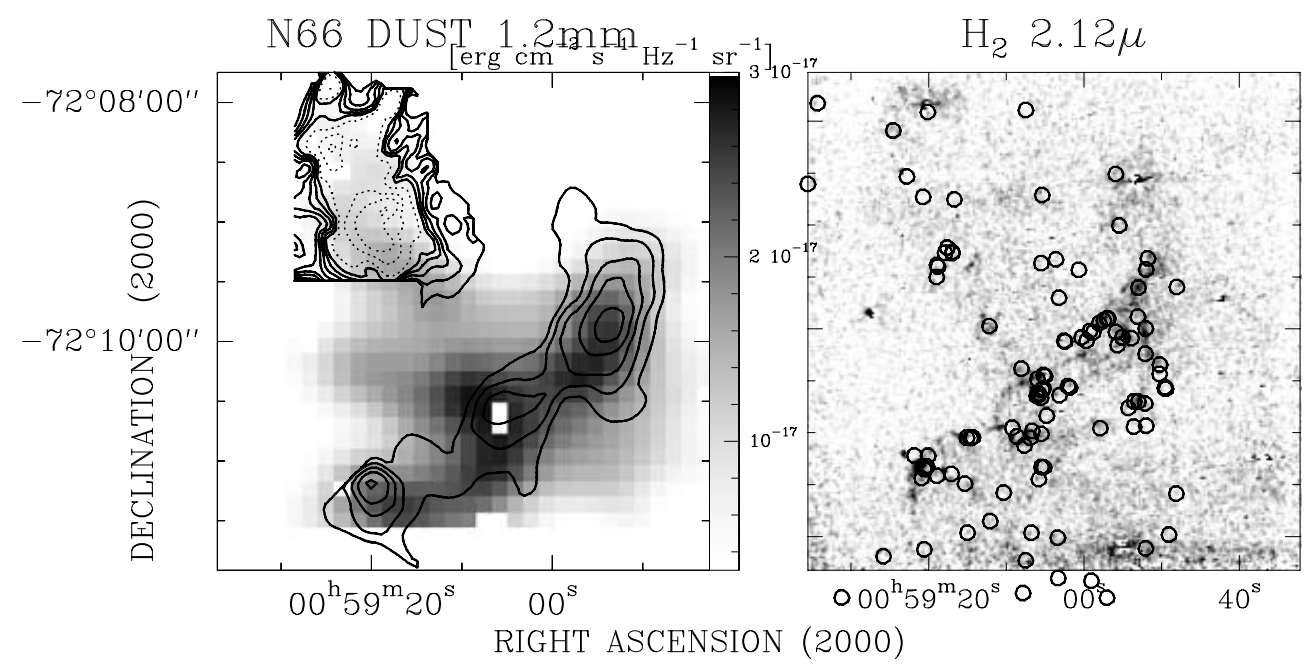

Figure 1. Left) The dust image at $1.2 \mathrm{~mm}$ of N66. Superimposed is the $\mathrm{CO}(2-1)$ emission line contours. Emission stronger than $2 \mathrm{~K} \mathrm{~km} \mathrm{~s}^{-1}$ is shown in dotted contours. Right) The $2.12 \mu \mathrm{m}$ $\mathrm{H}_{2}$ image of N66. The IR sources with J-Ks $>1.0$, plotted as open circles, follow the $\mathrm{H}_{2}$ emission and tend to concentrate towards the $\mathrm{H}_{2}$ knots.

SPITZER images (Bolatto et al. 2006) to undertake a study of the dust properties in these massive star forming regions. In this contribution we concentrate mainly on results obtained in N66 in the SMC and 30Dor in the LMC.

\section{The molecular gas}

There is existing evidence supporting that in low metallicity environments, due to an enhance photodissociation of the $\mathrm{CO}$ molecule, the gas mass derived from CO measurements could be underestimating the total amount of molecular hydrogen $\mathrm{H}_{2}$ (Rubio et al. 1993; Lequeux et al. 1994; Galliano et al. 2003). We have used the $1.2 \mathrm{~mm}$ millimeter dust emission to determine molecular masses and compared them to those obtained from $\mathrm{CO}$ observations. In the SMC, we have done this study in two regions, the bright HII region N66 (Perez 2006) and the SW region of the SMCBAR (Bot 2005; Bot et al. 2006).

In N66, we have combined deep NIR imaging with SPITZER 3.6, 4.5, 5.8, 8.0, 24, 70 and $160 \mu \mathrm{m}$, and $1.2 \mathrm{~mm}$ SIMBA observations, to determine the spectral energy distribution SED of the gas in the region and we have investigated the dust SED (Perez 2006).

\subsection{N66 in the $S M C$}

N66 is the brightest HII region in the SMC. It is powered by the central cluster NGC 346 , which contains $33 \mathrm{O}$ stars, of which 22 are earlier than 06 type. The distribution of the molecular emission as traced by the $\mathrm{CO}(2-1)$ emission line (Rubio et al. 2000) is shown in the contours in Figure 1a, and the molecular gas as seen in the molecular $H_{2}$ $2.12 \mu \mathrm{m}$ emission line in Figure 1b. Two distinct regions are identified in the CO contour map. One is the molecular gas associated to the bright optical filaments depicting the optical HII region and the molecular cloud to the north-east of the HII region. The CO emission of the HII region is very weak, while the northern cloud is bright in CO. In contrast, the $2.12 \mu \mathrm{m} \mathrm{H}_{2}$ emission is detected in the HII region with a hint of emission on the north-eastern spur. The molecular gas in the HII region is exposed to a strong 
UV radiation field from the $\mathrm{O}$ stars in the central core of NGC 346 as well as other $\mathrm{O}$ stars distributed in the area.

To determine the contribution of the dust emission to the measured SIMBA flux, we have determined the contribution due to the free-free emission in the region and that of the CO line. The latter is negligible and the free-free contribution represents $\sim 15 \%$ to the continuum $1.2 \mathrm{~mm}$ emission (Perez 2006). To determine the free-free contribution we calibrated a $\operatorname{Br} \gamma 2.16 \mu \mathrm{m}$ image of the region obtained with the $1.5 \mathrm{~m}$ Telescope at CTIO Observatory. We derived the free-free emission at $1.2 \mathrm{~mm}$ from the $2.16 \mu \mathrm{m}$ image by adopting a $\mathrm{T}=10^{4} \mathrm{~K}$, ionized region Case B (Reynolds 1992). We produced a derived free-free image at $1.2 \mathrm{~mm}$ at the SIMBA resolution and registered it to the $1.2 \mathrm{~mm}$ SIMBA image. We subtracted one from the other to obtain the dust emission image of N66 at $1.2 \mathrm{~mm}$, shown in Figure 1a. In this dust image we choose three different areas, two covering the HII region and one covering the northern-east molecular cloud. In these three areas we obtained the gas mass from the dust emission, assuming a dust to gas ratio of 0.10 for the SMC. In the same areas we obtained the integrated CO emission and determined the $\mathrm{N}\left(\mathrm{H}_{2}\right)$ column density of the gas using the Galactic conversion factor of $2.8 \times 10^{20} \mathrm{~mol} \mathrm{~cm}^{-2} \mathrm{~K}^{-1} \mathrm{~km}^{-1} \mathrm{~s}$ (Bloemen et al. 1986). The gas mass determined from the dust emission is larger by factor of 10 than that obtained from the $\mathrm{CO}$ emission in the massive star forming HII region while it is only a factor of 2.5 that of the quiescent north-east molecular cloud.

Using the N66 SPITZER images, plus the $2.16 \mu \mathrm{m}$ image and $2.12 \mu \mathrm{m} H_{2}$ image and the ISO CVF spectral images, it was possible to separate the SED due the contribution of the dust emission and that of the HII region. The HII and PDR-dust model (Boulanger, priv. comm.) cannot explain the measured dust emission at $1.2 \mathrm{~mm}$. To explain the $1.2 \mathrm{~mm}$ dust contribution to the SED a cold dust component is needed. The spatial extension of the dust is larger than the regions where CO is detected. The $160 \mu \mathrm{m}$ SPITZER data also shows a larger spatial extension than that of the CO clouds (Leroy et al. 2006). Thus, all these observations are consistent with large amounts of molecular mass in $\mathrm{H}_{2}$ gas envelopes and CO tracing only the dense region of the molecular cloud.

\subsection{The $S M C B A R$}

SIMBA $1.2 \mathrm{~mm}$ continuum observations were done toward the SMCBAR region. In this region, the gas mass derived from the dust emission was a factor of 10 or more larger than the mass determined from the virial mass determination using the $\mathrm{CO}$ emission for the cold quiescent cloud SMCB1-1 (Rubio et al. 2004). This unexpected result could only be explained if the cloud had a large $H_{2}$ gas envelope which the $\mathrm{CO}$ emission was not tracing. An extension of this study, where we combined all the observations done towards the SMCBAR region, was done by Bot 2005. The $1.2 \mathrm{~mm}$ SIMBA image is shown in Figure 2a. The dust $1.2 \mathrm{~mm}$ emission was obtained by subtracting the $\mathrm{CO}$ emission line contribution and the free-free emission at $1.2 \mathrm{~mm}$ deduced from published continuum radio observations. The dust emission was used to compute gas masses (Bot 2005, Bot et al. 2006). These gas masses were compared to virial masses determined from the CO emission, obtained from the $\mathrm{CO}(1-0)$ observations of the region done in ESO/SEST Key Programme: CO in the Magellanic Clouds (Rubio et al. 1993). The CO(1-0) emission line contours are overlaid in the SIMBA image in Figure $2 \mathrm{~b}$. The comparison of the gas mass determination for these GMC's in the SMCBAR showed that SMC cloud masses are systematically larger than the virial mass determination, confirming the result obtained in the SMCB1-1 cloud. Bot et al. (2006) conclude that CO is confined in clumps in large envelopes of $\mathrm{H}_{2}$ and that their motion is not representative of the gravitational potential and they suggest that the GMC's in the SMC are magnetically supported. 

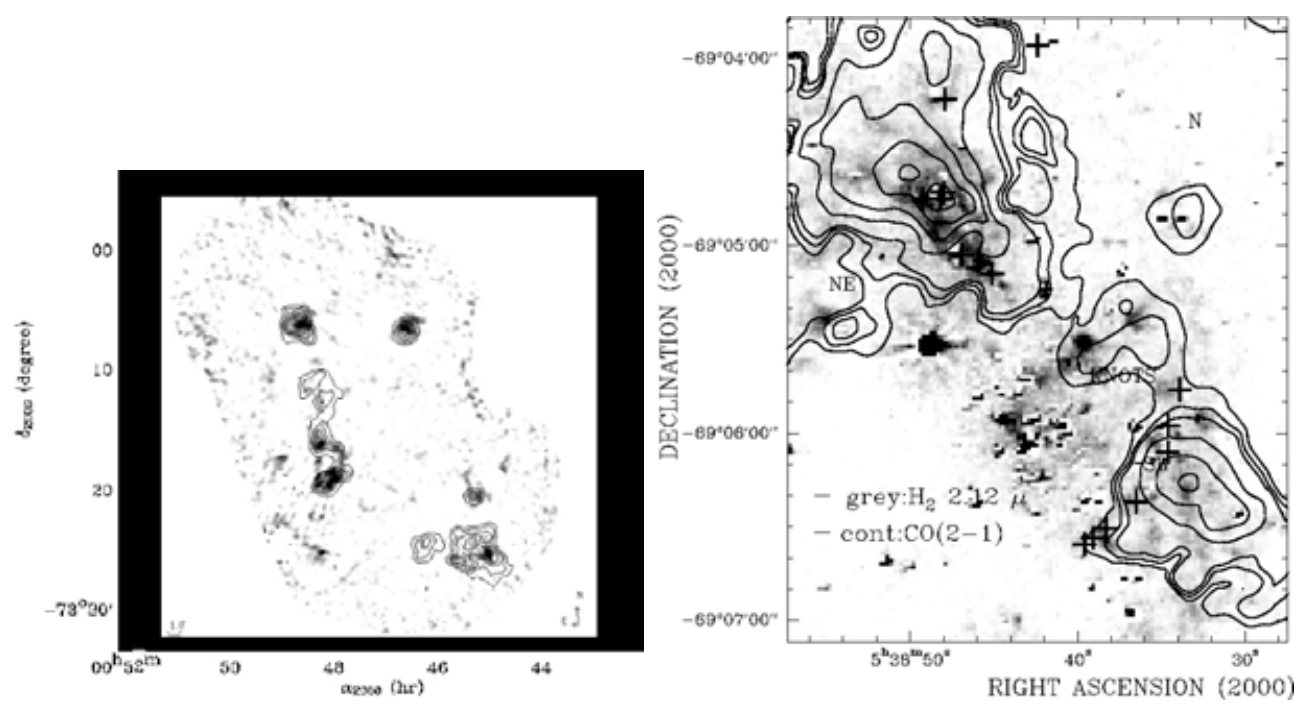

Figure 2. Left) The $1.2 \mathrm{~mm}$ SIMBA image of the SMCBAR with $\mathrm{CO}(2-1)$ contours superimposed. Right) The $2.12 \mu \mathrm{m} \mathrm{H}_{2}$ image of 30Dor. The $\mathrm{CO}(2-1)$ contours are superimposed. The IR embedded sources are plotted as black crosses.

\section{Massive star formation}

Deep JHKs images were obtained with the $2.5 \mathrm{~m}$ Dupont Telescope at LCO observatory of the central core NGC 346 and southern part of the HII region in N66. These images revealed very bright red sources, with $\mathrm{K}$ magnitude brighter than 13 and $\mathrm{H}-\mathrm{Ks}$ colors larger than 2.0. These embedded IR sources were found in the central NGC 346 cluster and in the $6.7 \mu \mathrm{m}$ ISO peaks $\mathrm{E}$ and $\mathrm{H}$ (Contursi et al. 2000) and maximum CO emission (Rubio et al. 2000). Their IR properties are those of massive young objects with large intrinsic IR excess, most probable Massive Young Stellar Objects (MYSO's) (Bik et al. 2006). IR spectra of two bright sources and one $H_{2}$ knot were obtained with SOFI/NTT at La Silla Observatory. The spectra show a rising continuum towards longer wavelengths and the series of $\mathrm{Br} \gamma$ lines and $H_{2}$ emission lines are detected, confirming their nature as MYSO's (Rubio \& Barbá 2006). In two spectra the He lines are present indicating a high temperature of the embedded source. These MYSO's represents a younger generation of massive stars than the $3 \mathrm{Myr}$ assigned to the NGC 346 cluster.

Nota et al. 2006. have found a rich population of pre-main sequence stars which are coeval with the massive central core NGC 346. This cluster concentrate the largest number of the pre-main sequence stars detected by them. The distribution of these stars concentrate towards the CO peaks in the HII region. Recent SPITZER observation of N66 evidence a large population of bright, very red infrared sources which are suggested to be YSO's based on their SED (Simon et al. 2006).

We have plotted in Figure $1 \mathrm{~b}$ the IR sources which have colors Ks-J $>1.0$ obtained and Ks $<15$ from NIR photometry on the distribution of the $2.12 \mu \mathrm{m}$ molecular hydrogen $H_{2}$ gas in N66. The $\mathrm{H}_{2}$ gas delineates the filaments of the HII region as seen in dark lanes in the HST image of Nota et al. (2006). These sources are located in the filaments of the $2.12 \mu \mathrm{m} H_{2}$ molecular gas and tend to concentrate near the $H_{2}$ knots. The confirmed MYSO's are located in the $\mathrm{H}_{2}$ knots. 


\section{30 Doradus}

30 Doradus is the brightest HII region in the LMC and has no counterpart in the Local Group. It is a giant HII region containing the nearest super star cluster (SSC) R136. The central cluster R136 has more than $65 \mathrm{O}$ stars with several WR concentrated in about $0.5 \mathrm{pc}$. This massive star forming region has two large giant molecular cloud in its surrounding. Weak CO emission coming from several dense clumps was detected in $\mathrm{CO}(2-1)$ between the two GMC's (Figure 2b). These dense clumps have masses of $10^{4} M \odot$ and they show a velocity gradient of several tens of $\mathrm{km} \mathrm{s}^{-1}$, consistent with expansion due to the kinetic energy of the winds from the massive stars in R136.

A second generation of massive stars was found in the border of the SW molecular cloud and in the NE one (Rubio et al. 1998) from NIR studies. These sources have magnitudes brighter than $\mathrm{Ks}=13$ and IR colors with $\mathrm{H}-\mathrm{Ks}>1.6$. Their IR properties are consistent with massive embedded sources from their positions in the color-color and color-magnitude IR diagrams. NICMOS/HST images towards these IR sources revealed that they were multiple sources in compact groups with several bright IR stars (Walborn et al. 1999).

Figure $2 \mathrm{~b}$ shows the distribution of the molecular $\mathrm{H}_{2} 2.12 \mu \mathrm{m}$ gas with the $\mathrm{CO}(2-$ 1) emission line contours superimposed. The $\mathrm{H}_{2}$ gas is clumpy and has a completely different morphology with respect to the ionized gas. Combining the $2.12 \mu \mathrm{m}$ image, a $2.16 \mathrm{Br} \gamma$ image, the ISO CVF data, and the SPITZER data, Boulanger \& Rubio (2006) have related the spatial and spectral distribution of the mid-infrared emission to the radiative and dynamical impact of the super star cluster R136 on the ISM structure and dust composition. They suggest that the spatial/clumpiness of the ionized and molecular gas is a key to understand the mid-IR emission of massive star forming regions. The surface filling factor of the molecular gas fixes the fraction of the stellar radiation which is absorbed in the PDRs. The density and the dust abundance in the HII gas fixes the fraction of Lyman continuum absorbed by dust rather than by $\mathrm{H}$ and He atoms. In 30 Doradus, the CO is arising from shielded interiors of dense clumps with a small surface filling factor (Poglitsch et al. 1995). In a clumpy medium, the ionizing photons are not expected to propagate ahead of the wind swept shell in the lower density gas and thus they could ionize the inter-clump medium. If so, then the ionization could increase the pressure at the surface of the dense clumps and may be produce infall, creating the conditions for a new burst of star formation.

In figure $2 \mathrm{~b}$ we have included the position of the brightest IR sources detected by Rubio et al. (1998). These concentrate towards the peak emission in $\mathrm{CO}(2-1)$ in the NE CO Cloud and projected over the bright $H_{2}$ gas concentrations. In the SW CO Cloud, the IR sources are located in the outer layer facing the R136 cluster where the CO emission shows a steep density gradient as if being compressed. The IR sources appear associated to $H_{2}$ knots where the PDRs are formed in the clumpy medium.

The general picture in this region points that new burst of massive star formation has occurred associated to the dense molecular clumps in the region.

\section{Conclusions}

We have found evidence from the dust emission in the SMC that the molecular gas $\mathrm{H}_{2}$ is more spatially extended than the $\mathrm{CO}$. In quiescent regions, the molecular gas mass is similar to a factor of two for $\mathrm{CO}, \mathrm{mm}$ and FIR determinations. In regions of massive star formation, the molecular gas mass determination can differ by an order of magnitude when derived from the dust mm emission, than when it is determined from $\mathrm{CO}$ or virial 
assumption. The molecular gas in massive star forming regions is clumpy with many PDR's regions associated to the dense molecular clumps. The molecular clouds in low metallicity environments seem to be mainly composed of $\mathrm{H}_{2}$.

In massive star forming regions, i.e, N66 in the SMC and 30 Dor and N11 in the LMC, studies reveal vigorous star formation in the surrounding of the massive central cluster. Embedded IR sources, $\mathrm{H}_{2}$ nebular knots, visible optical knots and O stars, and MYSO's are found associated to the dense clumps of molecular gas. The action of the UV radiation field and winds of the massive stars is interacting with remnants of the parental molecular cloud. Either by radiation pressure or by pushing the material through mechanical energy these stars contribute in generating the conditions for new star formation. However, the observations show that no massive cluster as the existing one is being formed. N66 could represent a case of triggered star formation as modeled by Elmegreen (1999).

Studies with SPITZER will be able to address questions such as which is the IMF of these new generation of stars, and the dust properties in low metallicity environments. ALMA, with its high resolution and sensitivity, will be the most suitable instrument to study the dense molecular clouds in the regions of massive star formation in the Magellanic Clouds.

\section{Acknowledgements}

We would like to acknowledge Dr. J. Palouš and Dr. B. Elmegreen for an excellent organization of the symposium. M.R. is supported by the Chilean Center for Astrophysics FONDAP No. 15010003.

\section{References}

Bik, A., Kaper, L. \& Waters, L. B. F. M 2006, A\& $A$ 455, 561

Bloemen, J. B. G. M., Strong, A. W., et al. 1986, A\&A 154, 25

Bolatto, A., Simon, J., Stanimirovic, S., et al. 2006, ApJ (Submitted)

Bot, C. 2005, PhD thesis, Universite Louis Pasteur, Strasbourg

Bot, C., Boulanger, F., Rubio, M. \& Rantakyrö, F. 2006, ApJ (Submitted)

Boulanger, F. \& Rubio M. 2006, in: L. Infante, M. Rubio \& S. Torres-Peimbert (eds.), Keys to Spitzer Observations of Luminous Star Forming Regions, RevMexAC (Instituto de Astronomia: UNAM), vol. 26 , p. 5

Contursi, A., Lequeux, J., Cesarsky, D., et al. 2000, A\&A 362, 310

Elmegreen, B. G., Kimura, T. \& Tosa, M. 1995, ApJ 451, 675

Fukui, Y., Mizuno, N., Yamaguchi, R., et al. 1999, PASJ 51, 745

Galliano, F., Madden, S. C., Jones, A. P., et al. 2003, A\&A 407, 159

Lequeux, J., Le Bourlot, J., Des Forets, G., et al. 1994, A\&A 292, 371

Leroy, A., Bolatto, A., Stanimirovic, S., et al. 2006, In preparation

Mizuno, N., Rubio, M., Mizuno, A., et al. 2001, PASJ 53, 971

Nota, A., Sirianni, M., Sabbi, E., et al. 2006, ApJ 640, L29

Perez, L. 2005, MSc thesis, Universidad de Chile, Santiago, Chile

Poglitsch A., Krabbe, A., Madden, S. C., et al. 2006, ApJ 454, 293

Reynolds, R. J. 1992, ApJ 392, L35

Rubio, M., Lequeux, J., Boulanger, F., et al. 1993a, A\&A 271, 1

Rubio, M., Barbá, R. H., Walborn, N., et al. 1998, A\&A 271, 1

Rubio, M., Contursi, A., Lequeux, J., et al. 2000, A\& $A$ 359, 1139

Rubio, M., Boulanger, F., Rantakyrö, F. \& Contursi, A. 2004, A\&A 425, L1

Rubio, M. \& Barbá, R. H. 2006, ApJL (Submitted)

Simon, J., Bolatto, A., Whitney, B., et al. 2006, in preparation

Walborn, N. R., Barbá, R.H., Brandner, W., et al. 1999, AJ 117, 225 


\section{Discussion}

ZINNECKER: Monica, would you agree that the big clusters that you have shown (NGC 346 and R136) do not trigger similar-sized new clusters in their neighboring clouds?

Rubio: Hans, according to the observational evidence on the new generation of stars forming in the region, there is no similar-sized clusters. The ones detected are compact groups with 3 or 4 massive YSO's but not as NGC 346 or R136. 